\title{
Use of a Microphone Phased Array to Predict Noise in a Ducted Tail Rotor
}

\author{
Qian Xiao ${ }^{1, a, *}$, Jun Huang ${ }^{1, b}$, Mingxu Yi ${ }^{1, c}$, Yacong $W u^{1, d}$ \\ ${ }^{1}$ School of Aeronautic Science and Engineering, Beihang University, College Road No.37, Beijing, \\ China

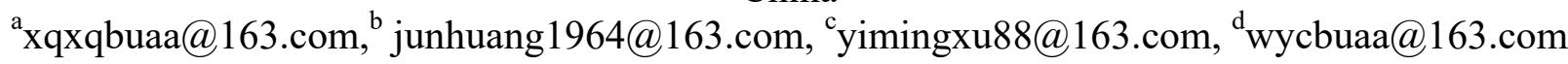

Keywords: ducted tail rotor, acoustic experiment, aerodynamic noise

\begin{abstract}
The aerodynamic noise experiment of the ducted tail rotor includes the hysteresis state and the former flight state. In recent years, the most commonly used and reliable test technology of aerodynamic noise prediction and control in aircraft - "Microphone phase array" measurement technology, which is use a large number of calibrated microphone to identify the wave front of the spatial sound source and use the same phase detection space acoustic field section, determining the distribution of source, and then research the noise propagation characteristics and control strategy of full-size model. At present, the aerodynamic experiment of helicopters is mainly focused on the main rotor and tail rotor and the acoustic test of the tail rotor is less. Therefore, this paper has concentrate on the aerodynamic noise test of the ducted tail rotor, which is of great significance to the development of it.
\end{abstract}

\section{Introduction}

Many experimental and theoretical studies contributed to a better understanding of the generated by ducted tail rotor. Researchers at home and abroad have carried out a lot of aerodynamic noise tests on helicopter rotor and tail rotor, including acoustic tests such as load noise, thickness noise and impulse noise of helicopters under different flight conditions, which mainly test in acoustic wind tunnel, silence room, outdoor site [1]. That is different test demands have different requirements for the test site. In recent years, France and Germany researchers have experimented with UH-1H rotor of US military, which proved the effectiveness of ONEAR and DLR proposed Euler/ Kirchhoff method to predict high-speed impulse noise in hover [2]. R.H.Schlinker and R.K.Amiet conducted a vortex disturbance noise measurement of the tail rotor in the acoustic study wind tunnel. David R.suttie et al. conducted orthogonal noise detection of the tail rotor and vortex in a low noise wind tunnel [3].

With the significant progress of sensors, data acquisition and computer processing technology, the use of microphone-phase array technology is becoming more and more extensive [4]. The aircraft industry heavily uses phased array for the identification of noise sources on engines, airframes, landing gear and other parts of an aircraft [5]. The ultimate goal of the present work is to use a microphone phased array for the ducted tail rotor, which is the first time in the domestic. 


\section{Laboratory Equipment and Model}

\subsection{Acoustic Wind Tunnel}

It is a single reflux low velocity and low turbulence acoustic wind tunnel used in this experiment, which own the opening and closing two replaceable test section, the rectangular cross- sectional area of the test section is $14 \mathrm{~m} \times 5.5 \mathrm{~m}$. The maximum wind speed of opening test section is $100 \mathrm{~m} / \mathrm{s}$, closed test section is $130 \mathrm{~m} / \mathrm{s}$. The open test section is mainly used for acoustic experiments with background noise of $75.6 \mathrm{db}$.

\subsection{Test Bench of Ducted Tail Rotor}

In the experiment, the diameter of the ducted tail rotor is $2 \mathrm{~m}$. The test bench is mainly composed of the foundation frame, the platform body, the power system and the blade system. Table 1 shows the main geometric parameters of the ducted tail rotor.

Table 1 The main geometric parameters of the ducted tail rotor.

\begin{tabular}{|c|c|}
\hline Geometric parameter & Size \\
\hline Paddle radius & $850 \mathrm{~mm}$ \\
\hline Airfoil of blades & NACA232012 \\
\hline Number of blades & 11 \\
\hline Center body diameter & $594 \mathrm{~mm}$ \\
\hline Spread angle & $4^{\circ}$ \\
\hline
\end{tabular}

\subsection{Test and Control System}

\subsubsection{Free Field Microphone in Far Field}

In order to measure the noise propagation characteristics of the ducted tail rotor model, 30 freefield microphones were arranged outside the inlet tail rotor inlet side in the test, as shown in Fig 1. A layer of sound absorption lining was laid in the support rod of free field microphone, to prevent the impact of acoustic reflection on the measurement results.

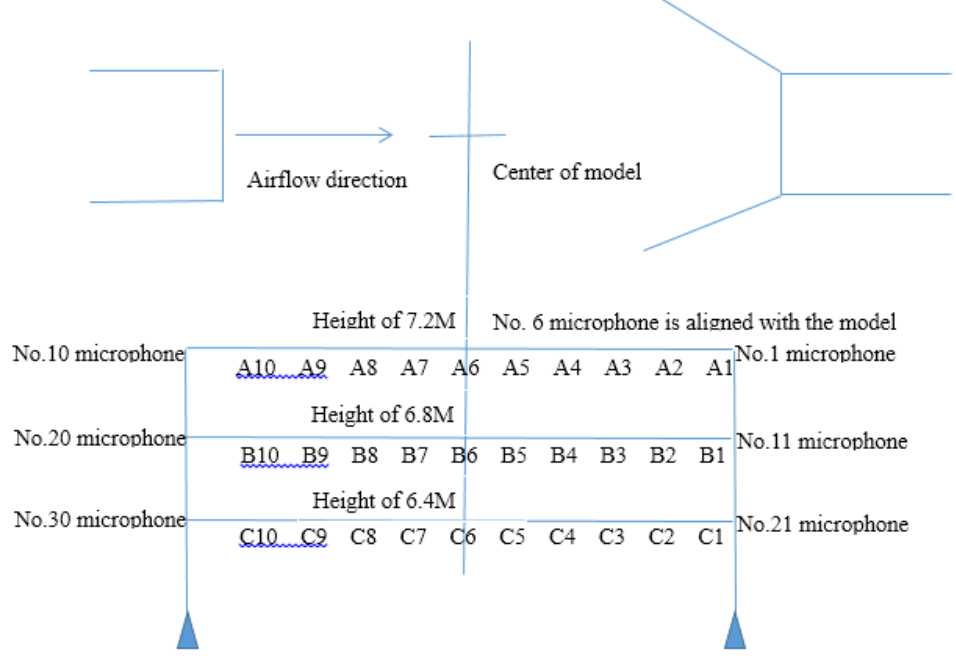

Figure 1 Microphone position layout. 
Adjacent microphone spacing is $0.8 \mathrm{~m}$ and the microphone support frame is $7.6 \mathrm{~m}$ from the center of the wind tunnel.

\subsubsection{Data Collection System}

The acoustic data acquisition system is a 272 channel dynamic data acquisition system. The highest sampling frequency of the system is $200 \mathrm{kHz}$, the model conversion bit is $24 \mathrm{bit}$, accuracy $\leq$ $0.10 \mathrm{~dB}$, dynamic measurement range $>120 \mathrm{~dB}$, anti-stacking protection $>110 \mathrm{~dB}$.

Test data acquisition using PXI bus data acquisition system. PXI bus data acquisition system is mainly composed of the pre-amplifier, data acquisition, communication cards, Kong's computer, data processing computer etc.

\section{Test Program and Methods}

The aerodynamic noise experiment of the ducted tail rotor includes the hovering state and the front flight state noise test, all in the opening test section, the test bench is installed on the ground through the transition stage. The free field microphone array is arranged on the inlet side of the ducted tail rotor model with a distance of $8 \mathrm{~m}$ from the paddle.

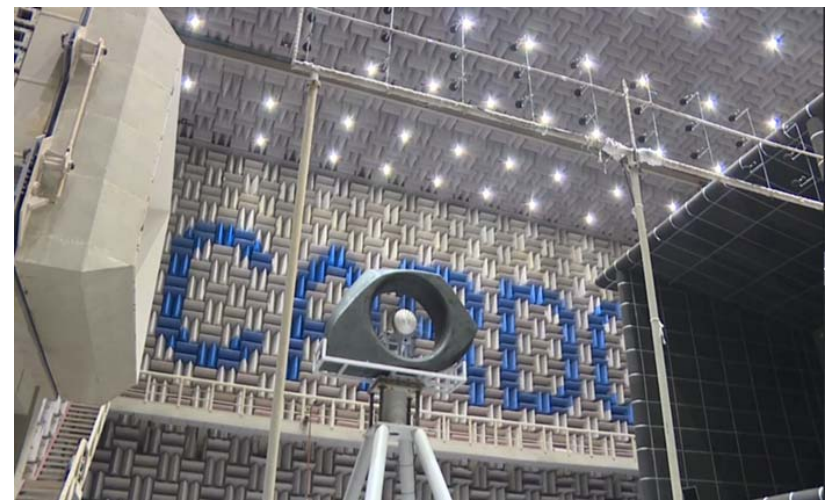

Figure 2 Test model of ducted tail rotor.

Firstly, installed the transition stage, and then model test bench was installed on the bench, measuring the model test bench noise under the flow conditions. Far field free field microphones were installed in the silencer room. After the model was installed in place, checking the installation of the model and the operation of the test bench to ensure that the model was installed accurately and the test bed could run correctly.

After the installation of the acoustic measuring equipment was completed, all the microphones were calibrated by the calibrator of the acoustic wind tunnel measuring system, and the sound pressure sensitivity, amplitude frequency and phase frequency curve of each microphone were obtained.

Before the start of the test, the transition stage, ducted tail rotor model test bench, far-field microphone support frame and other structures according to the needs of the surface to do silencer processing.

Confirmed the test equipment and model status, an acoustic test can be carried out. The speed of test bench was gradually increased to determine the safety. 


\section{Results and Discussions}

As the test for the helicopter in hysteresis state and the flight state, which mainly concerned about the aerodynamic noise characteristics of the tail rotor and research the noise when the ducted tail rotor in different states.

The aerodynamic noise of the ducted tail rotor at a location is analysed and compared with the case where the rotary speed is $\mathrm{n}=0,1300 \mathrm{rpm}, 1400 \mathrm{rpm}, 1500 \mathrm{rpm}$ and flight speed is $20 \mathrm{~m} / \mathrm{s}, 25 \mathrm{~m} / \mathrm{s}$, $30 \mathrm{~m} / \mathrm{s}$. In this paper, taking the test at A10 observation point as an example. The amplitude and phase of noise calculated by the test data analysis program are given in Figure 3 when the rotary speed is $1300 \mathrm{rpm}$ and free stream.
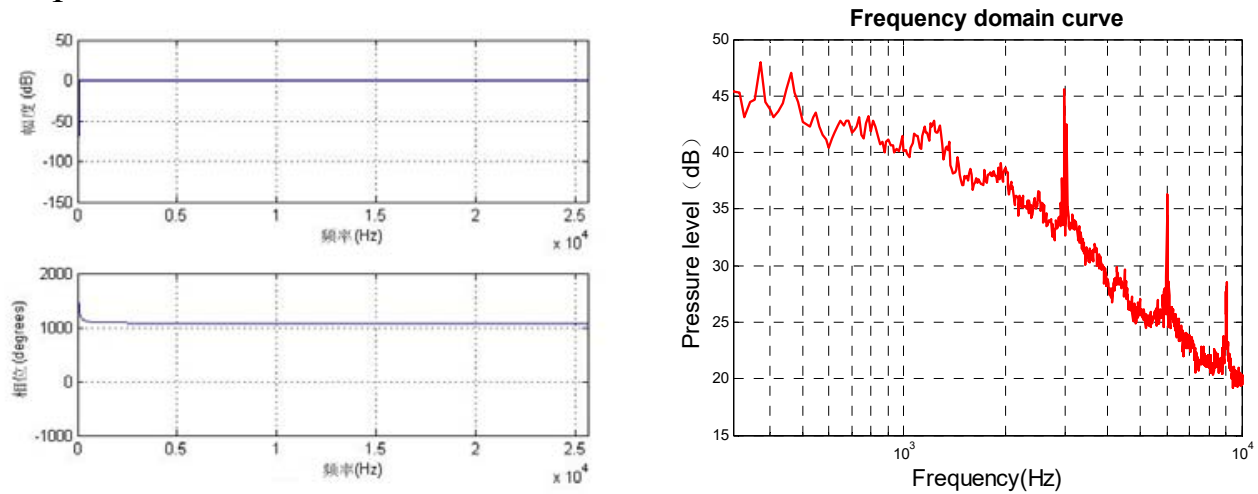

Figure 3 The amplitude and phase

Figure 4 Sound pressure level

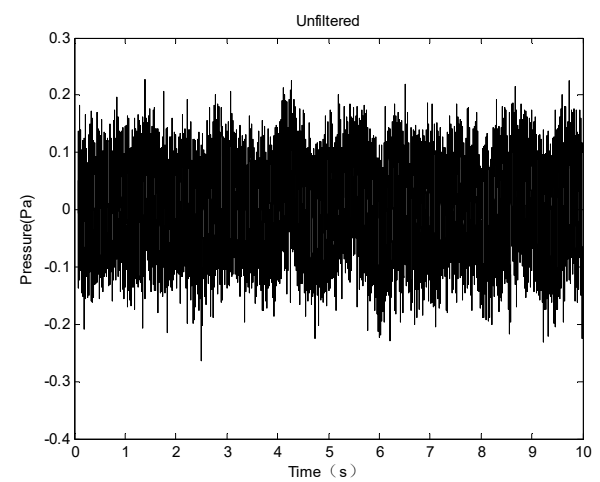

Figure 5 Unfiltered sound pressure

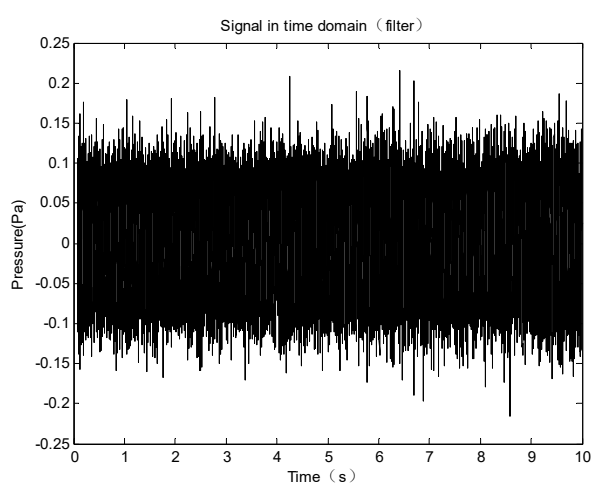

Figurer 6 Filter sound pressure

The pressure level is shown in Figure 7 and Figure 8 when rotary speed is 1400rpm, 1500rpm respectively.
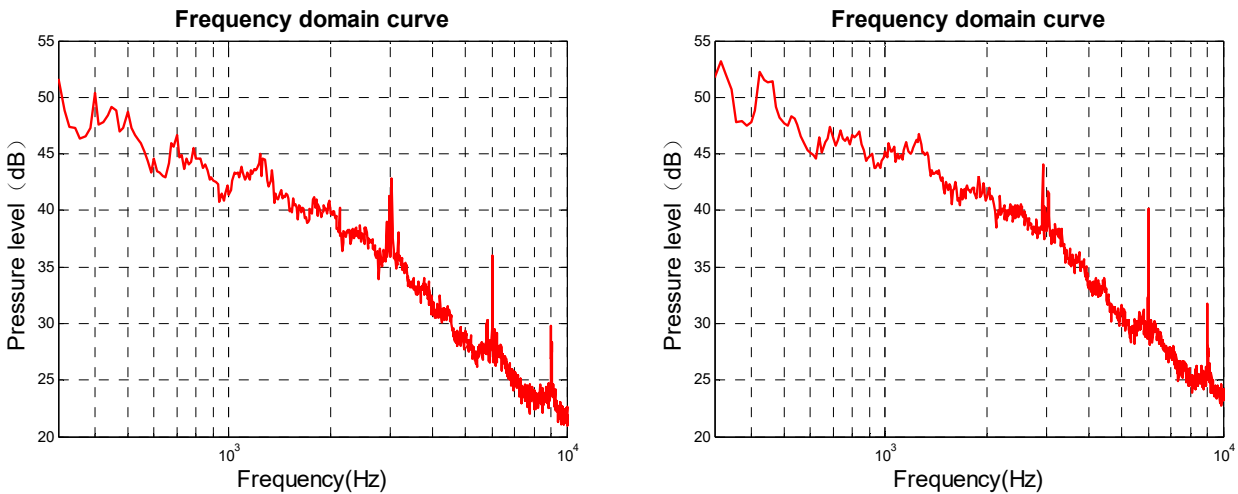

Figure 7 Sound pressure level at 1400rpm Figure 8 Sound pressure level at 1500rpm 
It can be seen from the figure that there are noise peaks in the low frequency band and the high frequency band. The noise peak of the low frequency band is generated by the rotation of the paddle tail rotor, mainly in the blade passing frequency (BPF) and its frequency multiplication, and the sound pressure level shows a decreasing trend with the increase of frequency order.

Table 2 The experimental results at different rotary speed

\begin{tabular}{|c|c|}
\hline Rotary speed(rpm) & Total sound pressure level(dB) \\
\hline 1300 & 65.3485 \\
\hline 1400 & 67.7364 \\
\hline 1500 & 70.1736 \\
\hline
\end{tabular}

The sound pressure level of the ducted tail rotor at 1300rpm rotary speed and different flight speed are shown in Figure 9-11, respectively.
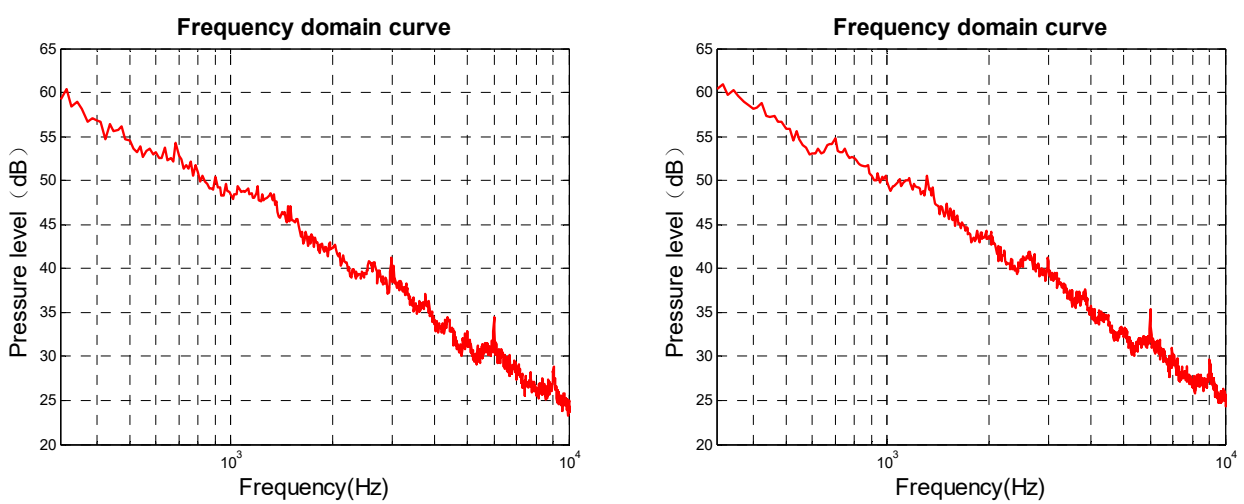

Figure 9 Sound pressure level at 20m/s Figure 10 Sound pressure level at $25 \mathrm{~m} / \mathrm{s}$

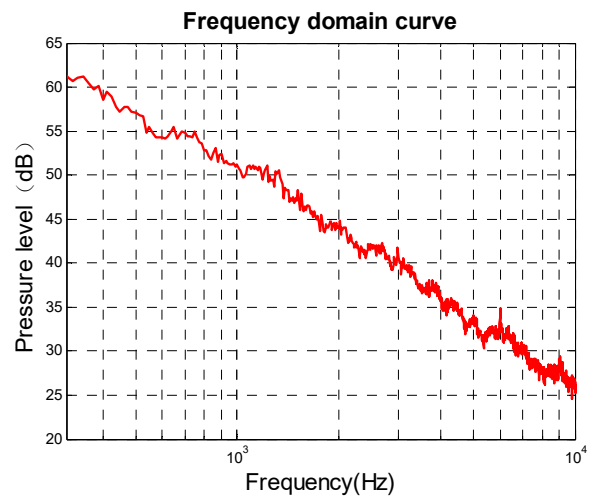

Figure 11 Sound pressure level at $30 \mathrm{~m} / \mathrm{s}$

The noise in flight state is mainly concentrated in the high frequency range above $1 \mathrm{kHz}$, the air flow and shear layer have a relatively great influence on the propagation path and amplitude of the noise.

Table 3 The experimental results at different flight speed

\begin{tabular}{|c|c|}
\hline Flight speed $(\mathrm{m} / \mathrm{s})$ & Total sound pressure level(dB) \\
\hline 20 & 76.5192 \\
\hline 25 & 77.7253 \\
\hline 30 & 78.8185 \\
\hline
\end{tabular}




\section{Summary and Conclusion}

In this paper, the microphone phase array measurement technology was applied to determine the aerodynamic noise of ducted tail rotor. The experimental result showed that the SPLs of the rotor in BPF and its harmonics increase with the increase of the rotary speed and flight speed. The results also showed that the SPLs of the rotor in BPF are higher than its harmonics.

\section{References}

[1] S. Khelladi, S. Kouidri, F. Bakir, R. Rey. Predicting tonal noise from a high rotational speed centrifugal fan. Journal of Sound and Vibration 313 (2008) 113-133.

[2] Ali Akturk, Cengiz Camci. Experimental and Computational Assessment of a Ducted-Fan Rotor Flow Model. Journal of Aircraft Vol. 49, No. 3, May-June 2012.

[3] A. S. Morgans, S. A. Karabasov, A. P. Dowling, T.P. Hynes. Transonic Helicopter Noise. AIAA Journal Vol. 43, No. 7, July 2005.

[4] D. Casalion, F. Diozzi, R. Sannino, A. Paonessa. Aircraft noise reduction technologies: A bibliographic review. Aerospace Science and Technology 12 (2008) 1-17.

[5] By Hee Dong LEE, Oh Joon Kwon. Detailed Aerodynamic Analysis of a Shrouded Tail Rotor Using an Unstructured Mesh Flow Solver. Transactions of the Japan Society for Aeronautical and Space Sciences. Vol. 47, No. 155, 2004. 